\title{
Clustering around latent variables approach to detect panel disagreement in three-way tea sensory evaluation
}

\author{
DR Fernando ${ }^{1}, S_{\text {Samita }}^{2}$, TUS Peiris ${ }^{3}$ \\ 1, 2 Postgraduate Institute of Agriculture, University of Peradeniya, Sri Lanka. \\ ${ }^{3}$ Bio-Statistics Unit, Faculty of Livestock Fisheries \& Nutrition, Wayamba University of Sri Lanka.
}

DOI: 10.29322/IJSRP.10.06.2020.p10291

http://dx.doi.org/10.29322/IJSRP.10.06.2020.p10291

\begin{abstract}
Sensory evaluation plays a remarkable role in maintaining the quality standards of beverages such as tea. The quality, which determines the price of tea, is evaluated by professional tea tasters. Uncertainty and vagueness of sensory evaluation has been a serious issue in selection of good quality tea. An issue existing when analyzing sensory data to detect panel disagreement is that data of three dimensional (three-way) or higher are often reduced to two-way data. Present study aimed to investigate the possibility of using Clustering around Latent Variables for three-way data (CLV3W) method to detect panel disagreement in sensory data of Sri Lankan tea. A three-way data set, 8 tea tasters $\times 13$ tea growing regions $\times 6$ attributes, for each month with four replicates (4 factories per region), for a period of one calendar year were used for the study. When CLV3W analysis was performed separately for each month data, it was found two-clusters (two-latent components) exist for the data. Attribute loadings of colour and strength indicated that they are represented by the first latent component, and those loadings for brightness, flavour, aroma, and quality indicated that they are represented by the second component. Region scores for the two components revealed grouping of regions indicating some regions have similar tea quality attributes. Assessor weights varied across latent components and months. However, by examining assessor weights it was possible to identify assessors those who were in agreement and those who were in disagreement for each component. This finding demonstrated the fact that certain assessors are more sensitive to certain attributes and thus in order to detect tea with certain attributes, appropriate assessors can be employed. Therefore, CLV3W method is a useful method to detect disagreement between assessors, especially in 3-way data, and it can effectively be used when selecting assessors for sensory evaluation.
\end{abstract}

Key words: Quality assessment, grouping attributes, higher dimensional array, disconsensus of tasters

\section{Introduction}

Food product quality is the leading factor that governs the food market. Since product quality and human responses to products are assessed in sensory evaluations, organoleptic properties play an important role in food quality concerns (Silva et al., 2014). Sensory evaluation is defined as a scientific method used to evoke measure, analyze, and interpret responses to products as perceived via the senses of sight, smell, touch, taste, and hearing (Tham et al., 2016). There are three commonly used sensory tests; discrimination test, descriptive test, and affective test. Discrimination test determines the sensory difference between samples, the descriptive test determines the nature or properties of the sensory difference, and affective test determines the intensity of preferences of the customers about the food (Tham et al., 2016). 
Role of sensory evaluation is emphasized in world famous beverages like tea (Kowalski et al., 2019). In Sri Lanka, tea is a major source of foreign exchange earnings. The quality of tea depends on the chemical components of tea infusions. The analytical methods available to identify chemical constituents impacting tea quality are precise. However, chemical composition analysis is complicated, time-consuming, labor-intensive, and expensive. Further, that process needs materials and a large amount of chemicals (Yin et al., 2018). As the sensory quality is a result of the interaction between the tea and the human sensation, instrumental assessments of organoleptic properties are dubious (Peiris et al., 2018). Traditionally, the quality of tea is assessed by professional tea tasters and interpreted in their own language. The tea industry relies on their assessments. As such, tea tasters play a crucial role in determining tea price (Kottawa-arachchi et al., 2014). The use of organoleptic evaluation to identify tea quality is rapid (Duan et al., 2018). Nevertheless, problems in human assessors like bias, the influence of external factors, change in the perceptiveness, and the time required to develop such professionals trigger ever-growing need for the monitoring of assessors (Don Francesco et al., 2014). Apart from overall monitoring, there may be special situations where it needs to identify the panelists who are more suitable to assess a certain set of attributes. Similarly, there may be situations where it is necessary to select a uniform set of panelists with outstanding performances, or to identify groups of panelists with similar levels of assessment. Then the requirement of statistical methods to assess an individual's reliability, discriminability, repeatability, selection of outstanding panel members and agreement with other members becomes important. Among those factors affecting assessing, this paper mainly concerns the identifying disagreement within a panel.

Many approaches of studying panel consensus have emerged in the literature. Consonance index (Dijksterhuis. 1995), Control of Assessor Performance (CAP) method (Schlich. 1996), egg-shell plot (Lea, Rødbotten, \& Næs, 1995) and linear mixed models (Latreille et al., 2006) are some of them. Due to its adaptability, analysis of variance (ANOVA) has been employed frequently. Carbonell et al., (2007) used ANOVA and principal component analysis (PCA), generalized procrustes analysis (GPA), cluster analysis and partial least squares (PLS) regression to evaluate panel performance. Panel concordance analysis (PANCA), which is an extension of PCA is also a tool that has been used to identify disagreement between the panelists (Derks. 2010). In another study, a parallel factor (PARAFAC) model (Romano et al., 2011) was used to investigate individual differences between assessors. The nway partial least squares (n-PLS) model was used to test the predictive ability of the panel (Romano et al., 2011). Assessor main effect in the classical assessor model of ANOVA describes the disagreement between assessors, and assessor $\times$ product interaction describes both panel disagreement and different use of scale (scaling effect) (Peltier et al., 2012). In some studies, a coefficient was used to down-weight the bad performance assessors (Tomic et al., 2013). The rationale behind this strategy has been to assign a unique weight to each assessor and thereby to identify good performers and bad performers. Rational methods like fuzzy logic, neural network, data aggregation, classification, and clustering have been proposed to solve the vagueness related to this field (Tham et al., 2016). Clustering of Latent Variables (CLV) which aimed at clustering sensory descriptors along with a latent component has been the strategy used in the study by Vigneau et al. (2003). As it aggregates the data over the assessors, information regarding the differences between assessors is lost.

Although many approaches have been proposed in the past for the analysis of scores given by assessors, the dimensionality of the data such as three-way have surprisingly been neglected. In most of the methods, data have been considered as two-way, such as product and assessors, attribute and assessors. In fact, most methods studied have considered each attribute separately, therefore relationships among the attributes have been ignored. Wilderjans \& Cariou. (2016) addressed this issue by extending CLV to CLV3W to disclose the underlying sensory dimensions. Present study aimed at using CLV3W method to detect panel disagreement in three--way sensory data of Sri Lankan tea. 


\section{Materials and Methods}

\subsection{The CLV3W method}

A conventional 3-dimensional sensory profiling data array $(\underline{\mathbf{X}})$ consists of scores given by $K$ assessors for $I$ products on $J$ sensory attributes (Wilderjans \& Cariou, 2016). In the CLV3W method, initially, $J$ attributes are grouped into $Q$ clusters and thereby determine $Q$ latent components; $t_{1}, t_{2}, \ldots, t_{Q}$. Then, for each component, attribute loadings, product scores and assessor weights are estimated. Panel disagreement is evaluated using cluster-specific assessor weights $\left(\mathbf{w}_{\mathrm{q}}\right)$, which are determined by maximizing the function (Wilderjans \& Cariou, 2016).

$\mathrm{g}=\sum_{\mathrm{j}=1}^{\mathrm{J}} \sum_{\mathrm{q}=1}^{\mathrm{Q}} \mathrm{p}_{\mathrm{jq}} \operatorname{cov}^{2}\left(\mathbf{X}_{j} \boldsymbol{w}_{q}, \mathbf{t}_{q}\right)$

where

$\mathbf{X}_{j}-j^{\text {th }}$ lateral slice of $\underline{\mathbf{X}}$ and $\mathbf{X}_{j}$ is of order $I \times K$

$p_{j q}$ - Indicator whether attribute $j$ belongs $\left(p_{j q}=1\right)$ or does not belong $\left(p_{j q}=0\right)$ to cluster $q$.

This is equivalent to minimizing the loss function (Wilderjans \& Cariou, 2016).

$f=\sum_{j=1}^{J} \sum_{q=1}^{Q} p_{j q}\left\|\mathbf{X}_{j}-\boldsymbol{\alpha}_{j q}\left(\mathbf{t}_{q} \mathbf{w}_{q}^{\prime}\right)\right\|_{F}^{2}$

where

$\boldsymbol{\alpha}_{j q}$ - loading of the attribute $j$ in cluster $q$.

The CLV3W model is a special case of the cluster-wise PARAFAC model where there are $Q$ clusters of attributes and only one latent component is associated with each cluster. That latent component is described with attributes in that particular cluster. Generally, in the cluster-wise PARAFAC model, the elements of one of the three-dimensions (products, attributes and assessors) of sensory data array are assigned to a limited number of mutually exclusive clusters. Simultaneously, the data within each cluster are modeled with PARAFAC. In the conventional PARAFAC method, components are sought that capture the complex interaction between the elements of the three-dimensions. Then parameters of matrices of each dimension and components are estimated. These parameter estimates are made for each element of each dimension for a particular component. Loadings are the estimates of attributes. They reflect the extent to which the attributes represent the underlying components. Product scores are the estimates of product dimension and they indicate the location of each product on these components. Using product scores of different components, a grouping of products can also be investigated and thereby products with the same attributes can be determined. Estimates of assessors are called weights, which represent the extent to which the assessors rely on a particular component. Assessor weights are used to determine the panel disagreement (Wilderjans \& Ceulmans. 2013). In CLV3W, the principle used is exactly the same as of cluster-wise PARAFAC except one latent component is sought for each cluster and component-wise estimates for elements of three-dimensions are computed.

\subsection{Algorithm of CLV3W analysis}

Algorithm used in CLV3W is discussed by Wilderjans and Cariou (2016). In implementing this algorithm to the present study, agglomerative hierarchical clustering (AHC) was used for the initial partitioning as recommended by them. The number of clusters were decided by evaluating the difference of loss function (delta) values resulted in agglomeration steps. The number of clusters at the step that gives the largest delta is used as the basis of deciding the number of clusters. Attribute loadings, product scores, and assessor weights are estimated for each identified cluster. However, since the aim of the study was to detect the disagreement, the scaling 
effect was removed by using isotropic scaling factor adjustment (Wilderjans \& Cariou, 2016) before performing the analysis. The analysis was performed using functions in R-package of ClustVarLV.

\subsection{Application of CLV3W}

This CLV3W method was applied to the secondary data of Sri Lankan tea tasters used in the study Peiris et al. (2018). Data set contained scores given by 8 well-experienced tea tasters for samples from 13 tea growing regions of Sri Lanka on 6 sensory attributes. Each region had been replicated 4 times by means of 4 factories per region and data had been collected over a one-year period on a monthly basis, January to December of 2009. Since only one value per region is required for the CLV3W analysis, the median value of the 4 factories was used as the value of the region. Scores had been assigned on a 5-point rating scale. In fact, such a scale was advocated by several authors within the scope of data exploration (Cariou \& Wilderjans. 2018). The 13 regions considered in the study were, Nuwaraeliya (NE), Udapussellawa (UP), Lindula/Thalawakelle (LT), Agarapathana (AGP), Bogawanthalawa $(B G W)$, Maskeliya (MSK), Malwatta Valley (MWV), Bandarawela (BW), Uva Medium (UM), Mid Country (MC), Sabaragamuwa $(S G)$, Mataral Akuressa (MA), Galle/Alpitiya (GA) and the sensory attributes considered were colour, brightness, strength, flavour, aroma, and quality. Data of assessor 1 and 3 were not available for the month of September. The CLV3W analysis was performed separately for each month.

\section{Results}


The delta values resulted in agglomeration steps are presented in Figure 1 and it can be seen that the sharpest increase of the delta lies at the aggregating step two clusters to one, suggesting two-cluster or two-component solution for attributes. Moreover, the aggregation patterns in figure 1 were consistent across all months and thereby two-component solution was confirmed.

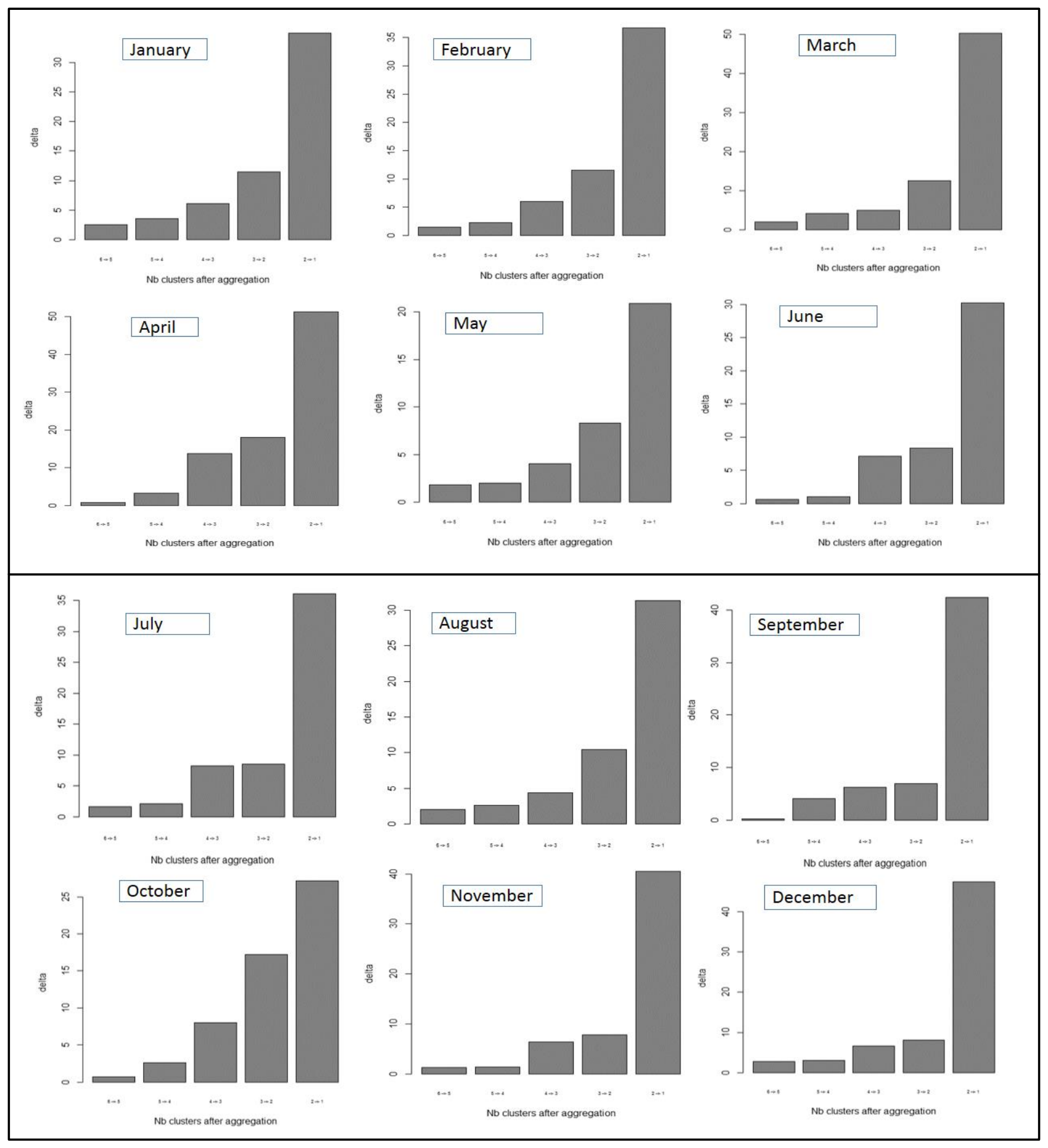

Figure 1: Evolution of delta with number of clusters for months January to December.

Loadings of attributes for each of the two components are presented in figures 2(a) and 2(b). From figure 2(a) it can be seen that only colour and strength have non-zero loadings and thus those two are the indicators of component one. Similarly, according to figure 
2(b), brightness, flavour, aroma, and quality are the indicators of component two. A surprising clustering was observed for the month of April where the first latent component consisted of only colour and second of all other attributes. Note that the loadings of the month April are not included in fig 2 as the loading of colour for component 1 was unusually large (9.31) thus the inclusion of that could hinder the general pattern revealed by all other months. Further, it can be noticed that loadings for all attributes in each component have the same sign, either positive or negative and it implies that attributes in each component are positively correlated. In all months, loadings of colour are higher than that of strength in component one, and brightness showed the lowest loading in component two. This can be interpreted as, the attribute colour has a higher contribution to the latent component one, and brightness contribution was the lowest to component two. Also, loadings for all attributes of both components in January, May, and July are negative and this confirms the fact that all attributes of both components are negatively correlated. Further, all attributes of both components are positively correlated in August, September, October and December where loadings for both components are positive.

(a)

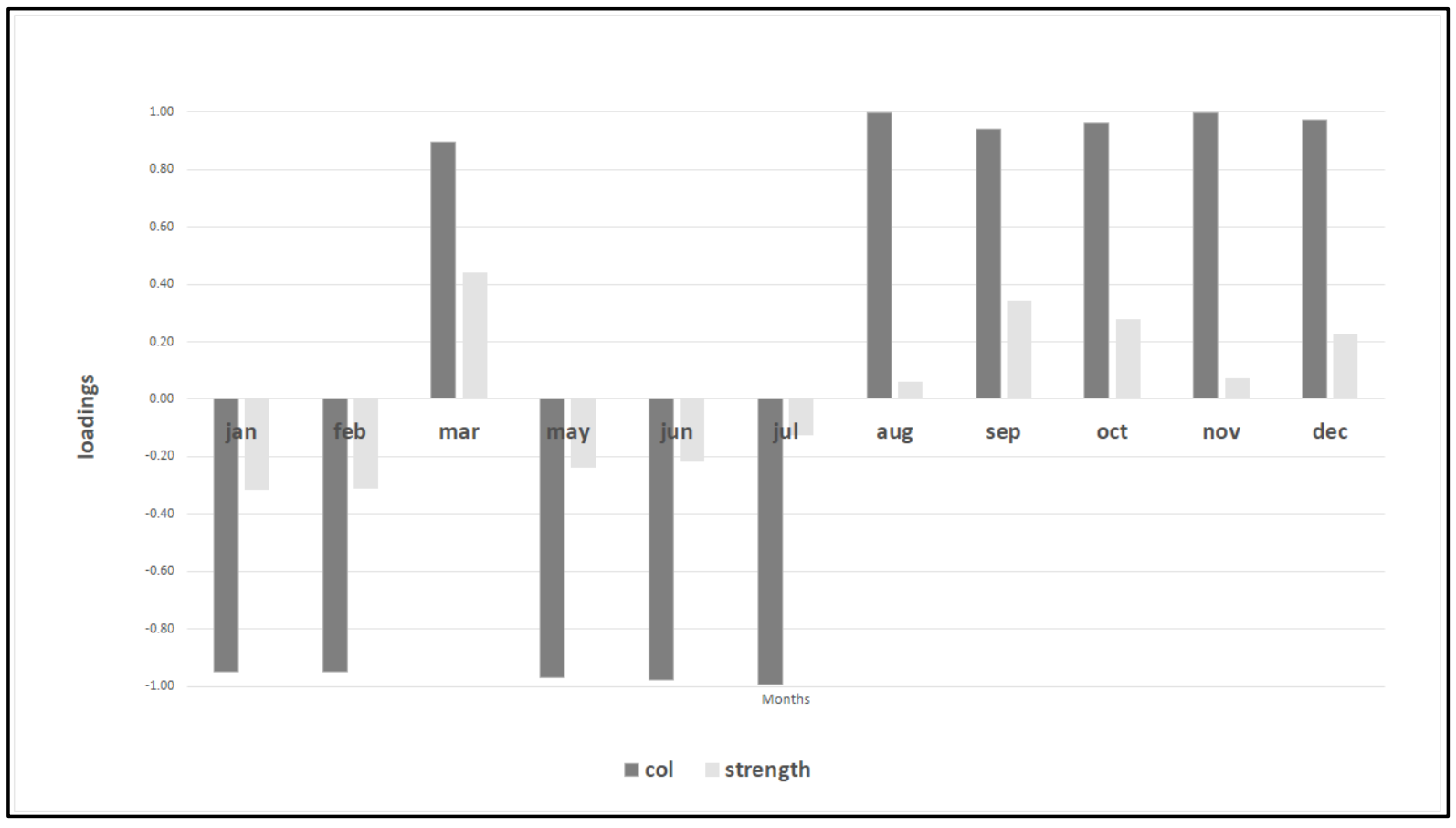


(b)

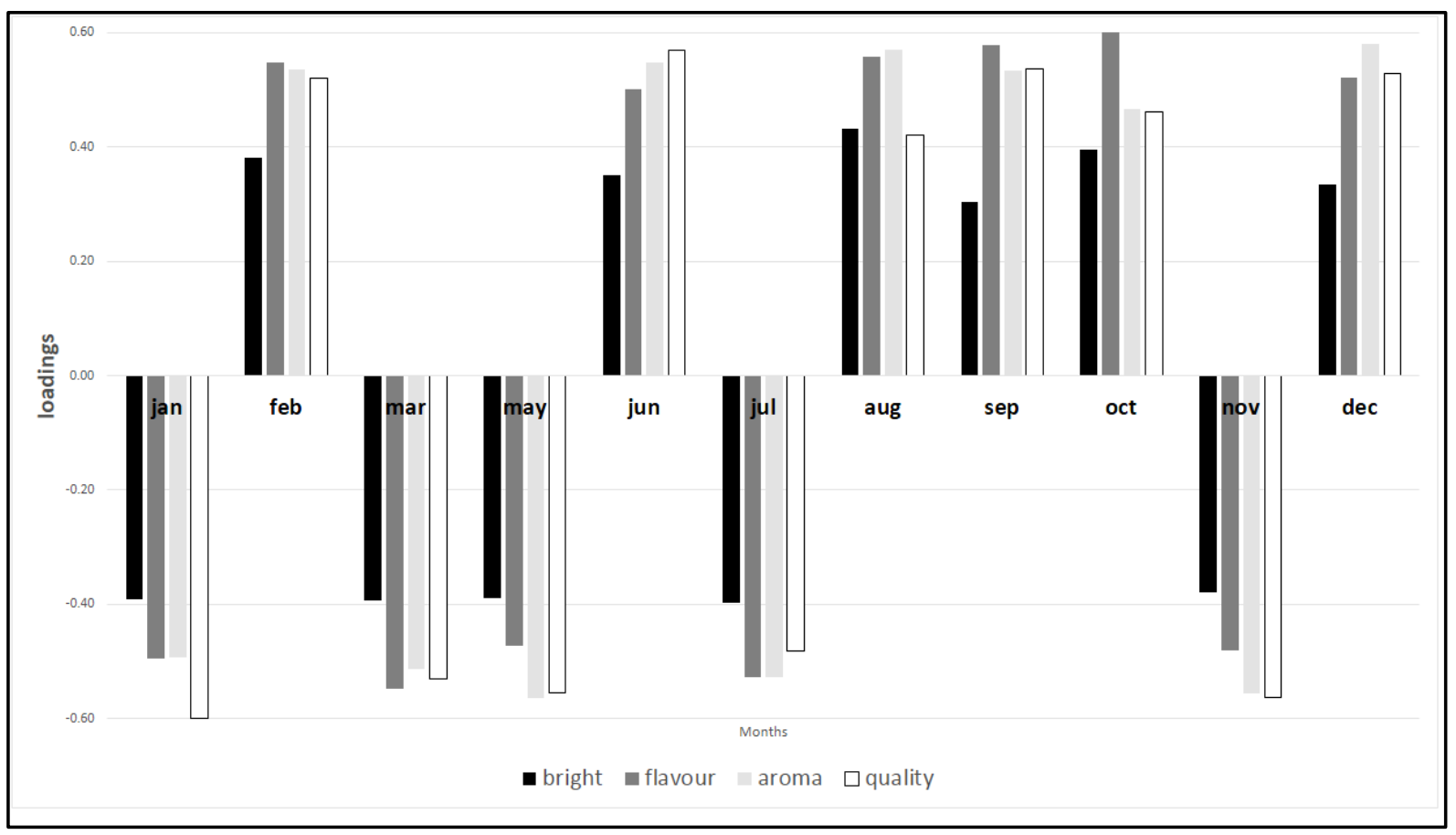

Figure 2: Loadings of attributes for clusters; (a) Component 1 (b) Component 2

The location of regions based on scores of the two components for each month is presented in figure 3. In all months, regions $N E$ and $U M$ were clearly disjointed from all other regions. Furthermore, some regions located close to each other indicating those regions have more or less same tea quality attributes (Circled in plots of figure 3). According to figure 3, by considering all months we may identify mainly 2 groups of regions as follows; $A G P, B G W, M S K$, and $L T$ in one group and $G A, M A$, and $S G$ in another group. 


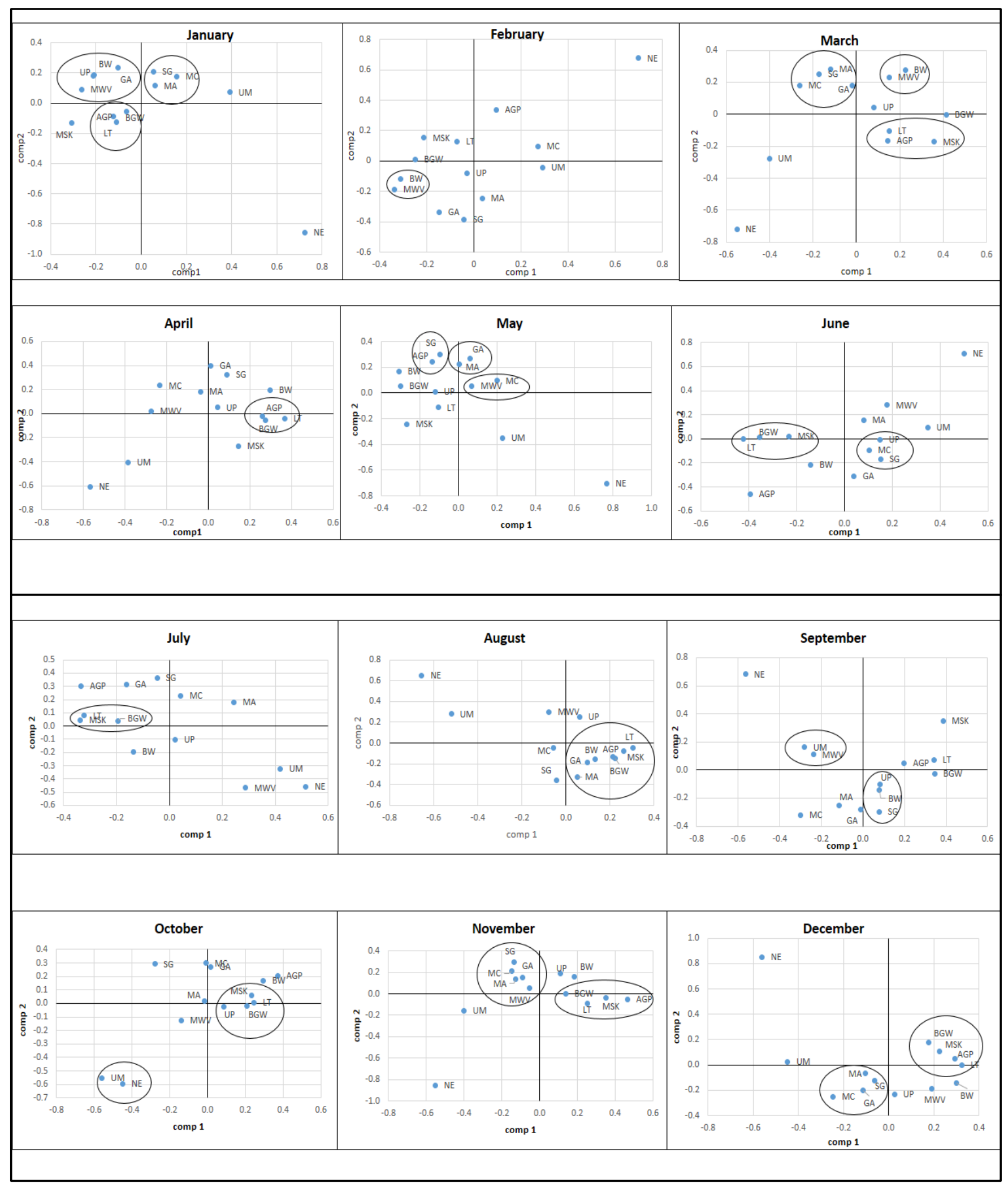

Figure 3. Configuration of region scores for two components in each month 
Assessor weights are the key parameters that determine panel disagreement. Since assessor weights are estimated for each component separately, disagreement can be studied with respect to each component. The assessor weights obtained for the two components for each month are presented in figure 4 . From figure 4 it can be seen variations of weights across latent components and assessors.
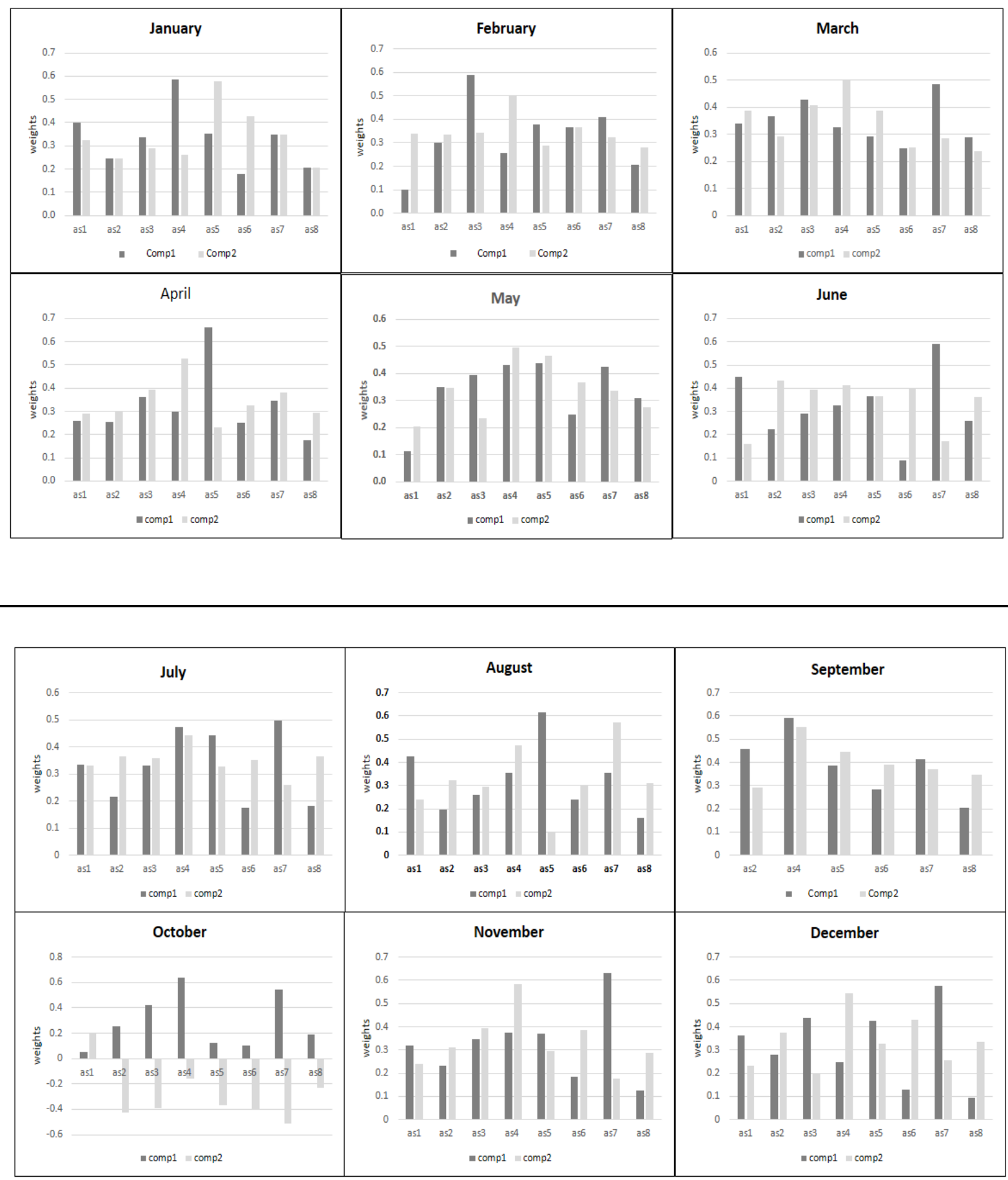
In order to have a better understanding of assessors who are in agreement for each component, assessors who had weights higher than the average weight for each component and month were tabulated (Table 1). From the table, it can be found that assessors 5 , 4 , 7 and 3 are generally in an agreement for the component 1 and thus they are more suitable to assess attributes of colour and strength. Similarly, assessors 4, 6 and 5 are in agreement in assessing latent component two, and thus they are more suitable to evaluate attributes brightness, flavour, aroma and quality. Since assessors 4 and 5 are in both groups, those two suitable to assess all 6 attributes.

\begin{tabular}{|c|c|c|c|c|c|c|c|}
\hline \multirow{2}{*}{$\begin{array}{l}\text { Month } \\
\text { January }\end{array}$} & \multirow{2}{*}{$\begin{array}{l}\begin{array}{l}\text { Latent } \\
\text { Component }\end{array} \\
1\end{array}$} & \multicolumn{6}{|c|}{$\begin{array}{l}\text { Assessors with above } \\
\text { the average weights }\end{array}$} \\
\hline & & & 3 & 7 & 5 & 1 & 4 \\
\hline & 2 & & & & 7 & 6 & 5 \\
\hline \multirow[t]{2}{*}{ February } & 1 & & & 6 & 5 & 7 & 3 \\
\hline & 2 & & & & & 6 & 4 \\
\hline \multirow[t]{2}{*}{ March } & 1 & & & & 2 & 3 & 7 \\
\hline & 2 & & & & 5 & 3 & 4 \\
\hline \multirow[t]{2}{*}{ April } & 1 & & & & 7 & 3 & 5 \\
\hline & 2 & & & & 7 & 3 & 4 \\
\hline \multirow[t]{2}{*}{ May } & 1 & 6 & 2 & 3 & 7 & 4 & 5 \\
\hline & 2 & & & 2 & 6 & 4 & 5 \\
\hline \multirow[t]{2}{*}{ June } & 1 & & & 4 & 5 & 1 & 7 \\
\hline & 2 & 5 & 8 & 3 & 6 & 4 & 2 \\
\hline \multirow[t]{2}{*}{ July } & 1 & & 3 & 1 & 5 & 4 & 7 \\
\hline & 2 & $\begin{array}{ll}5 & 1\end{array}$ & 6 & 3 & 2 & 8 & 4 \\
\hline \multirow[t]{2}{*}{ August } & 1 & & & 7 & 4 & 1 & 5 \\
\hline & 2 & 3 & 6 & 8 & 2 & 4 & 7 \\
\hline \multirow[t]{2}{*}{ September } & 1 & & & 5 & 7 & 2 & 4 \\
\hline & 2 & & & 7 & 6 & 5 & 4 \\
\hline \multirow[t]{2}{*}{ October } & 1 & & & & 3 & 7 & 4 \\
\hline & 2 & & 5 & 3 & 6 & 2 & 7 \\
\hline \multirow[t]{2}{*}{ November } & 1 & & 1 & 3 & 5 & 4 & 7 \\
\hline & 2 & & 5 & 2 & 6 & 3 & 4 \\
\hline \multirow[t]{2}{*}{ December } & 1 & & & 1 & 5 & 3 & 7 \\
\hline & 2 & & 5 & 8 & 2 & 6 & 4 \\
\hline
\end{tabular}

In fact, conventional cluster analysis was also performed on weights of assessors for each month and each component to ratify the above results. The majority of dendrograms exhibited the same cluster patterns as presented in table 1 . This result also supported the findings on the agreement among assessors as found using CLV3W method.

\section{Discussion}

Sensory evaluation plays a very important role in the tea industry as outcome from the sensory evaluation is the primary factor in determining the price of the product. In fact, in a country such as Sri Lanka, tasters can have an impact on the whole economy as tea is a major source of foreign exchange earnings to Sri Lanka. This emphasizes the necessity of monitoring the tea tasters regarding the 
individual's reliability, discriminability, and consensus with other tasters, and selection of uniform panel members depending on the set of attributes. Focusing on the latter criterion, the objective of present study was to identify the disconsensus if any, among a panel of tea tasters, and recently developed three-way analysis method was used to accomplish it. In the CLV3W method, out of the estimates; attribute loadings, region scores, and assessor weights, using assessor weights, the agreement between assessors can be determined.

Two clusters of attributes were the solution for all months and thus the 6 attributes can be seen as two independent sets of attributes. This result can be taken as a true representation as this outcome is not just following a particular clustering algorithm. This result in fact, was obtained as the best outcome based on delta (figure 1) by considering all possible random initial partitioning of attributes in the rational Agglomerative Hierarchical clustering. Except for April, the clustering pattern was the same for all months. We suspect the unusual pattern in the month of April could be due to some errors in recording the data. January, February, May, June, and July presented negative loadings for the component 1 and it could be interpreted as those attributes, colour and strength were not prominent during those months. Similarly, during four months; January, March, May, and July showed negative loadings for component 2 indicating those attributes; brightness, strength, flavour, and aroma were not prominent during this period. It should be noted that CLV3W method grouped sensory attributes according to their covariances, either positive or negative, with the latent component of each cluster. Romano et al., (2011) evaluated a panel performance using two-component PARAFAC model and by reanalyzing the same study, Wilderjans and Cariou (2016) reported that loadings of attributes in CLV3W seemed to be an oblique rotation of the PARAFAC axes. The PARAFAC method does not facilitate rotation, and in addition, interpretation of loading is much easier in CLV3W method as loading of the attributes that are not associated with a component is set to zero. The CLV3W method provides information not only with respect to the level of agreement between assessors but also about tea growing regions with similar attributes. The latter information is a striking outcome from CLV3W method for panel leaders. In fact, this study paved the way to identify tea growing regions with; poor on both groups of attributes, good in only one group of attributes and poor in the other group of attributes, and good in both groups of attributes. This is crucial information when marketing tea. Further, this result can be used to identify the panel's discriminability of regions for a particular group of attributes.

Romano et al., (2014) developed a multivariate assessor model, an extension of the univariate multiplicative assessor model with several attributes. Although this method can handle 3-way arrays, this method focuses only on differences of scale in different uses and not the disagreement between assessors. Likewise, the clustering of assessors can be done as clustering of consumers (Onwezen et al., 2012), but the resulting partitions then depend only on the chosen attribute. In 2013, Tomic et al. proposed performance indices to detect assessors of off-performance which is useful for panel leaders. However, they have recommended the method only as a complementary tool and never alone (Tomic et al. 2013). Alternatively, some authors advocated the use of fuzzy cluster analysis techniques (Shinde et al., 2017) as these methods enjoy appealing properties such as fuzzy membership and flexibility. However, the understanding and applications of the fuzzy theory are still scarce in the field of sensory data (Tham et al., 2016). Pinto et al., (2014) suggested the use of Cronbach's alpha (CA) coefficient as an analytically simple method to identify panelists familiar with welldefined attributes and rank the panelists as their consensus. However, this method also has limited use as it has not been modified for multivariate panel performance evaluation. Although the coefficient principle was later improved as a unique weight, which reflects the assessor's overall agreement with the rest of the panel, it ignores the fact that assessors may agree with the panel on one latent component and can totally disagree with another component. The weighting strategy in CLV3W was developed to address this point. 
Assessor weights for each latent component are important when discriminating tea samples of different regions. Higher and similar weights derived for a group of assessors on a particular component suggests that they are in agreement of assessing tea samples representing that particular latent component. In other words, such a group has the expertise in assessing attributes representing that particular component than attributes representing other components. Assessors who are not listed corresponding to each component and month in table 1 had lower weights for that component and month. This implies that those assessors' expertise is lower to capture the attributes of that component than other assessors who are in agreement. If weights are different among all assessors, then it can be suggested that panelists are not in agreement at all. However, such a total disagreement was not found in this study. It can be suggested that assessors with low weights need more training on relevant components. In fact, panel leaders can use CLV3W weighting strategy effectively to identify assessors who need more training on which subset of attributes. In situations where panel leaders need to identify a uniform set of panelists with outstanding performances, assessor weights of CLV3W method would be an ideal choice. These are paramount interests of sensory evaluations undertaken by tea tasting companies, and CLV3W method can provide the required information to those companies.

\section{Conclusion}

Identifying suitable assessors for sensory evaluations is a challenge and the CLV3W method provides an effective solution to this challenge, especially for sensory data with three-way arrays. Component-wise assessor weights can be used to identify panel disagreements. In this study, it was found that assessors 5, 4, 7 and 3 were generally in an agreement for the attributes colour and strength, and they are more suitable to identify tea with those attributes, or to evaluate samples from tea growing regions where those attributes are expected to be prominent. Similarly, assessors 4, 6 and 5 were found to be more suitable to identify tea with attributes, brightness, aroma, flavor, and quality. It seems, these assessors have differences in competence and thus, each group can be employed for a specific purpose. Therefore, CLV3W method is an option to detect disagreement as well as grouping of assessors. It can be suggested that weights of well-trained assessors derived by CLV3W method can be used as an index in fitting models to determine the price of different food that relies on assessor or consumer evaluations.

\section{Acknowledgment}

Authors thank Dr. Veronique Cariou, research lecturer at Statistics, Sensometrics and Chemometrics Unit, Nantes University, France.

\section{References}

Carbonell, L., Izquierdo, L., \& Carbonell, I. (2007). Sensory analysis of Spanish mandarin juices. Selection of attributes and panel performance. Food quality and preference, 18(2), pp.329-341.

Cariou, V., \& Wilderjans, T. F. (2018). Consumer segmentation in multi-attribute product evaluation by means of non-negatively constrained CLV3W. Food Quality and Preference, 67, pp.18-26.

Derks, E. P. (2010). PANCA: Panel concordance analysis. Food quality and preference, 21(3), pp. 324-329.

Di Donfrancesco, B., Gutierrez Guzman, N., \& Chambers, E. (2014). Comparison of results from cupping and descriptive sensory analysis of Colombian brewed coffee. Journal of Sensory Studies, 29(4), pp.301-311.

Dijksterhuis, G. (1995). Assessing panel consonance. Food Quality and Preference, 6(1), pp.7-14. 
Duan. Y, Song X, Yang Y, Li L, Zhao, Y, Wang, Y, Fang, W., \& Zhu, X. (2018). Correlation between Sensory Evaluation Scores and Flavor Attributes of Black Teas from Around the World. Journal of Food Processing \& Technology, 9:4

Kottawa-Arachchi, J. D., Gunasekare, M. T. K., Ranatunga, M. A. B., Punyasiri, P. A. N., Jayasinghea, L., \& Karunagodad, R. P. (2014). Biochemical characteristics of tea (Camellia L. spp.) germplasm accessions in Sri Lanka: correlation between black tea quality parameters and organoleptic evaluation. IJTS.

Kowalski, R., Wyrostek, J., Kałwa, K., Kowalska, G., Pankiewicz, U., Sujka, M., \& Mazurek, A. (2019). Sensory and chemical evaluation of tea brews prepared with the assistance of ultrasound. Ciência Rural, 49(11).

Lea, P., Rødbotten, M., \& Næs, T. (1995). Measuring validity in sensory analysis. Food Quality and Preference, 6(4), pp.321-326.

Latreille, J., Mauger, E., Ambroisine, L., Tenenhaus, M., Vincent, M., Navarro, S., \& Guinot, C. (2006). Measurement of the reliability of sensory panel performances. Food Quality and Preference, 17(5), pp.369-375.

Onwezen, M. C., Reinders, M. J., van der Lans, I. A., Sijtsema, S. J., Jasiulewicz, A.,Dolors Guardia, M., et al. (2012). A cross-national consumer segmentation based on food benefits: The link with consumption situations and food perceptions. Food Quality and Preference, 24(2), pp.276-286.

Romano, R., Vestergaard, J. S., Kompany-Zareh, M., \& Bredie, W. L. (2011). Monitoring panel performance within and between sensory experiments by multi-way analysis. In Classification and Multivariate Analysis for Complex Data Structures, (pp. 335-342). Springer, Berlin, Heidelberg.

Romano, R., Næs, T., \& Brockhoff, P. B. (2014). Combining analysis of variance and three-way factor analysis methods for studying additive and multiplicative effects in sensory panel data. Journal of Chemometrics, 29(1), pp.29-37.

Peltier, C., Brockhoff, P. B., Visalli, M., \& Schlich, P. (2012, July). Monitoring panel performances with the Mixed Assessor Model. Meta-analysis of the SensoBase. Peiris, T.U.S., Walgampaya, C.K., Thattil, R.O, Banks, D.L, \& Abeysinghe, I.S.B. (2018). Use of Mandel's bundle of lines model to improve agreement of a panel of tea tasters. J National Science Foundation Sri Lanka, 46 (1), pp.95-102.

Pinto, F. S. T., Fogliatto, F. S., \& Qannari, E. M. (2014). A method for panelists' consistency assessment in sensory evaluations based on the Cronbach's alpha coefficient. Food Quality and Preference, 32, pp.41-47.

Schlich, P. (1996). Defining and validating assessor compromises about product distances and attribute correlations. In Data handling in science and technology, (Vol. 16, pp. 259-306).

Shinde, S., Halder, K., Kumar, N., Meena, G. S., \& Pathera, A. K. (2017). Physico-chemical characterization and fuzzy logic modeling of sensory evaluation for market Ghewar. IJCS, 5(6), pp.1809-1816.

Silva, A. Y. S. L., Binduhewa, A. M. C. U., \& Subodinee, A. A. M. (2014). A study to recruit and train the product oriented sensory panel. International Journal of multidisciplinary Studies, 1(2).

Tham, H. J., Tang, S. Y., Teo, K. T. K., \& Loh, S. P. (2016, June). A review on intelligent sensory modelling. In IOP Conference Series: Earth and Environmental Science, (Vol. 36, No. 1, p. 012065). IOP Publishing.

Tomic, O., Forde, C., Delahunty, C., \& Næs, T. (2013). Performance indices in descriptive sensory analysis-A complimentary screening tool for assessor and panel performance. Food Quality and preference, 28(1), pp.122-133

Vigneau, E., \& Qannari, E. M. (2003). Clustering of variables around latent components. Communications in Statistics-Simulation and Computation, 32(4), pp.11311150 . 
Wilderjans, T. F., \& Ceulemans, E. (2013). Clusterwise Parafac to identify heterogeneity in three-way data. Chemometrics and Intelligent Laboratory Systems, 129, 8797.

Wilderjans, T. F., \& Cariou, V. (2016). CLV3W: A clustering around latent variables approach to detect panel disagreement in three-way conventional sensory profiling data. Food quality and preference, 47, pp.45-53.

Yin, X., Xu, X., Zhang, Q., \& Xu, J. (2018). Rapid determination of the geographical origin of Chinese red peppers (Zanthoxylum Bungeanum Maxim.) based on sensory characteristics and chemometric techniques. Molecules, 23(5), 1001.

\section{Authors}

First author: Ms. D. R. Fernando, MSc. student, Postgraduate Institute of Agriculture, University of Peradeniya, Sri Lanka. rajni0131@ outlook.com

Second author: Prof. S. Samita, Senior Professor, Postgraduate Institute of Agriculture, University of Peradeniya, Sri Lanka. ssamita@pdn.ac.lk

Third author: Dr. T. U. S. Peiris, Senior lecturer, Bio-Statistics Unit, Faculty of Livestock Fisheries \& Nutrition, Wayamba University of Sri Lanka. turslap@gmail.com

\section{Corresponding author:}

Ms. D. R. Fernando, Postgraduate Institute of Agriculture, University of Peradeniya, Sri Lanka rajni0131@outlook.com

+94710661438 